\title{
The art versus the science of medicine. Are clinical practice guidelines the answer?
}

Clinical practice guidelines (CPGs) are systematically created recommendations to assist physicians in the decision making processes related to the choice of health interventions. ${ }^{12}$ There has been increasing interest in the development of CPGs within all medical fields. In 1993 there were 374 publications indexed by MEDLINE as pertaining to CPGs; by 1996, the number had increased to over 980. The American Medical Association has listed in their "Directory of Medical Parameters" over 1600 sets of guidelines. $^{3}$ The enthusiasm and frenzy are such that "Guidelines for the development of guidelines" have been proposed. ${ }^{4}$ The American College of Rheumatology has also developed several sets of guidelines covering areas such as osteoarthritis, rheumatoid arthritis, and steroid induced osteoporosis..$^{5-11}$

Variation in practice has been reported for most fields in medicine, including musculoskeletal diseases, often driven by physician preferences or practice characteristics. For instance, prescriptions of second line drugs for patients with rheumatoid arthritis vary among rheumatologists. ${ }^{12} 13$ Demographic characteristics of the prescribing physician seem to have more impact in the selection of specific second line agents than the clinical characteristics of the patient. ${ }^{13}$ Similar variation has been seen at the primary care level and other specialties for osteoarthritis and low back pain. ${ }^{14-18}$ In one study where outcomes were reported patients with low back pain treated by "high prescriber" family physicians had the same outcomes as patients treated by "low prescribers", but much higher medical costs. ${ }^{17}$ In this age of shrinking budgets and limited resources practice variation may be considered inappropriate not only when resulting in differences in outcomes (benefits or risks) but also when substantial economic costs arise from interventions with unclear advantages.

Where there is no clear evidence favouring the use of one drug over another, practice variation may be readily understandable. Physicians frequently make decisions that are not evidence-based, often because there is no firm evidence on which to base them. They perform via "rules of thumb"- "medical heuristics". ${ }^{19}$ Decision making processes and "rules of thumb" may have been learnt at medical school or be based on subsequent experience, often with small numbers of patients. Guidelines can be merely "rules of thumb" in another guise especially when based largely on expert opinion. We all need principles and standards for clinical decision making - that is what medical school is all about-but, can these be improved by the use of CPGs, and should these be prescriptive?

At face value, the idea of having fast uniform "cookbook" responses for every medical problem is appealing. Proponents of CPGs believe that practice can be standardised to provide the best possible care. Adherence to CPGs could eliminate inappropriate variation, improve outcomes and decrease costs, through the reduction of ineffective health services. Yet, uniformity in practice styles should not be perceived as an end on its own, but as a means to improve patient outcomes and system efficiencies. Despite these potential benefits and the initial enthusiasm (mainly from policy makers and administrators), the expanding dissemination and addition of CPGs to mainstream medical practice is viewed with considerable scepticism and as a threat to autonomy (mainly by clinicians). ${ }^{2021}$
How are guidelines different from systematic reviews or expert consensus? Systematic reviews of interventions entail ongoing searches of the literature pertinent to the area of interest following a methodical approach (comprehensive electronic searches, hand searching of bibliographies and journals, etc). The available information is then classified according to pre-determined methods to evaluate the degree of scientific validity, with controlled clinical trials as the benchmark. Finally, if certain assumptions are met, findings from various sources may be pooledsynthesised quantitatively-(meta-analysis). The Cochrane Collaboration is a worldwide organisation with a mandate to produce systematic reviews of health interventions, ${ }^{22}$ but it does not produce guidelines. Consensus methods on the other hand are based on strategies that create structured environments in which experts provide levels of agreement on unresolved, and often controversial subjects. Guidelines are statements about practice developed following an evidence-based approach, expert recommendations, or a combination of both. If the evidence for a particular clinical scenario is solid-that is, several randomised clinical trials with comparable results-excellent CPGs can be developed that should readily be accepted by most. In this situation however, practice variation may be more limited and eventually, many physicians may adopt, albeit gradually, the new evidence into their practice. When the evidence for particular interventions is absent or weak, variation can understandably occur. An example of such scenarios is illustrated by the early use of second line drugs in patients with rheumatoid arthritis where it is well recognised that many treatments are more effective than placebo, ${ }^{23}$ and that early administration produces a better response..$^{24}{ }^{25} \mathrm{We}$ found in our utilisation studies that second line therapy was given early and with the same frequency by both communitybased and academic rheumatologists in our health region. ${ }^{126}$ On the other hand, significant variation was seen in the use of particular second line drugs, possibly reflecting the lack of evidence to strongly support one drug over the others. ${ }^{23}$

Financial advantages and reimbursement incentives may result in inappropriate physician practices. ${ }^{27}$ Economic profit may increase the utilisation of some interventions with no clear patient benefit. Although we believe that most physicians are decent human beings who care for the well being of their patients, we also recognise that economic incentives have important effects on human behaviour. Inappropriate practice can therefore be expected if personal financial gains are substantial, in particular if there are no important harms to patients (for example, over utilisation of innocuous diagnostic tests). Yet, it is unclear how guidelines can change this pattern. If the motivation behind particular practices is economic, physicians may not modify their behaviour after a set of recommendations has been introduced. If the incentive is financial, the most effective disincentive will also be financial, well beyond the scope of CPGs for physicians.

Can guidelines be useful? In our view they could be, if developed under certain guidelines! Systematic reviews of scientific evaluations of clinical guidelines have shown controversial results. ${ }^{28} 29$ Grimshaw reported that CPGs could improve both the process of care and patient outcomes, but with wide variation in their effect size. ${ }^{2}$ On 
the other hand, recent reviews found no evidence that CPGs produced significant benefits in clinical outcomes. ${ }^{28} 29$ In some instances nevertheless, the implementation of guidelines may be driven by economic considerations in contrast with beneficial patient outcomes. What attributes make CPGs worth the effort?

\section{Issue to be tackled}

To have a significant impact, the condition for which guidelines are developed should be prevalent or costly, and inappropriately managed, including either a wide use of ineffective or harmful interventions, or practice variation resulting in differing outcomes or costs.

\section{Clear evidence}

The ultimate goal of clinicians is to improve patient outcomes, and CPGs developed solely on the basis of financial considerations should not be accepted by the medical community. Clear evidence of efficacy must be available before recommendations can be endorsed. ${ }^{30}$ This evidence should be based in most instances on the results of randomised clinical trials. If economic aspects are the major driver for guideline implementation, it should be clear at the very least that there will not be a deleterious effect on patient outcomes. Yet, even in the presence of solid evidence, the dissemination of new medical knowledge is a slow process. Antman ${ }^{31}$ showed that interventions that could have saved lives were not adopted by experts in their recommendations until 10 years after the benefits had been clearly shown. How CPGs could improve this slow process is not clear. Given that experts seem to be as slow as practitioners in accepting new evidence, adding a new layer of scrutiny and evaluation with panels and task forces could possibly delay the process even more. The potential causes for this resistance to adopt new evidence are beyond the scope of our commentary. However, if evidence-based results are met with resistance, then merely opinions, even if from experts, can be expected to elicit more controversy. In our view, CPGs developed by expert panels in the absence of clear scientific evidence should be subjected to the same degree of scrutiny as any other intervention, and be tested in clinical settings before their wide implementation and adoption.

\section{Scope}

Parsimony is the key. Evidence is often clear for specific issues but insufficient to tackle the whole picture. Guidelines could be developed recommending the best dose and administration route to begin treatment of rheumatoid patients with methotrexate. It is unlikely nevertheless that evidence-based guidelines could be established for the treatment of rheumatoid arthritis in general. This is too broad a scope, including interventions as dissimilar as diagnostic tests, drugs, surgery and rehabilitation, and patients with varying degrees of severity and disease duration. The Agency for Health Care Policy and Research (AHCPR) from the US Department of Health spent millions in the past decade to develop CPGs. Among others, they produced "Clinical Practice Guidelines for the Management of Acute Low Back Pain". ${ }^{32}$ The scope of these guidelines includes "strategies for the assessment and treatment of acute low back problems in adults" by primary care physicians. The review of the literature was exhaustive, categorised into levels of evidence for recommendations. The document produced by AHCPR is an excellent systematic review on the current knowledge for the treatment of low back pain. It also includes guidelines for every aspect of care, from physical examination to referrals to surgeons presented, unfortunately, as complex graphical algorithms transporting patients across various decision nodes. The guidelines have been broadly distributed and published in North America. We were particularly interested in their recommendations on the initial use of lumbar radiographs in patients with low back pain, based on the recognition that they are seldom useful, and should only be considered if tumour, fracture or infection are suspected. The panel proposed a series of "red flags" or markers that could possibly identify patients with these disorders. "Red flags" included age, corticosteroid use, previous cancer, etc. We applied these criteria to over 960 consecutive patients with acute low back pain seen in primary care clinics. ${ }^{33}$ Lumbar radiographs had been requested in $13 \%$ of the patients; if physicians had followed the AHCPR guidelines, use would have increased to $44 \%$ ! These findings point to the need to evaluate CPGs in clinical settings before their wide dissemination, particularly if developed by consensus in the absence of clear data. In our view, the systematic review conducted by the AHCPR low back pain panel is scientific, comprehensive, and serves the medical community well. The guidelines however are too broad in their scope. An alternative approach would have been to identify two or three areas where inappropriate practice can be shown, where the evidence for an intervention is robust, the target patient population can be recognised, and complex algorithms are not required; for instance, prolonged bed rest is harmful for most patients with back pain, more costly than encouraging patients to maintain their level of activity, and is still widely recommended by some physicians. Unfortunately, much of the physician resistance to CPGs relates to the publication of bad guidelines, or recommendations with such a wide scope that they cannot be specifically applied to individual patients.

\section{Physician targets}

CPGs are often targeted at primary care physicians because it is clearly difficult to keep abreast of new knowledge for all the conditions seen in primary care, and it is perceived that simple rules may facilitate care. Many guidelines, such as those produced by the American College of Rheumatology, target specialists. Of more interest to us is not the "type of physician" but the "type of human being" who could benefit from guidelines. Rogers has shown that the diffusion of innovations follows striking sinusoidal patterns. ${ }^{34}$ People can be categorised according to their enthusiasm in adopting new technologies as: (a) innovators (venturesome); (b) early adopters (respectable); (c) early majority (deliberate); (d) late majority (sceptical); and (e) laggards (traditional). These categories apply to fields as different as the use of herbicides by farmers, clothing fashions, and yes, medical practice. Socioeconomic status, personality, and communication behaviour relate to the various "adopter" categories. The challenge is to be able to modify inappropriate behaviour through diffusion of interventions (guidelines or other) in people with varying behaviours. "Late majority" and "laggards" may benefit from evidence gathered locally or incentives to change traditional practice patterns. "Innovators" and "early adopters" may need some restraint before embracing new technologies with unconfirmed benefits.

\section{Development strategies}

Guidelines have higher rates of success when developed internally (in contrast with nationally or internationally), with input from potential users, and involving opinion leaders respected by their peers. ${ }^{35}$ Guidelines developed by a single national organisation (for example, American College of Rheumatology) cannot possibly consider the implications of varying patient populations, socioeconomic factors, and health costs for communities across the world. 
Many of the diseases treated by rheumatologists vary in prevalence or prognosis across ethnic populations. Many procedures taken for granted in North America are not accessible for most patients in developing countries. Relative costs of health interventions also vary across countries as shown for everyday treatments such as non-steroidal anti-inflammatory drugs. ${ }^{36}$ Local groups may not be able to undertake systematic reviews and CPG developments that are costly and time consuming. An alternative may be for local groups to select guidelines produced by national or international agencies and tailor them according to their needs, emphasising their involvement in the implementation phases.

\section{Dissemination strategies}

Specific educational interventions and continuing medical education have higher chances of success. Publication in journals have below average probability of being effective. ${ }^{2}$ Yet, most CPGs are just published in journals, and seldom formally implemented. Identifying those physicians who may benefit from specific interventions and being selective in their implementation (that is, "laggards" versus "innovators") may be more effective than a universal approach such as guideline diffusion in medical journals. More research is needed to evaluate physician behavioural changes with different dissemination strategies, including the effect of individual characteristics and beliefs on the success or failure of specific interventions.

Clinical practice guidelines should be considered as one more tool in the challenge to improve patient care. Are guidelines the solution for every medical dilemma? Evidently not. Some policy makers embrace CPGs as the solution to all problems. Unfortunately, most difficult questions in clinical medicine remain difficult because there is no clear answer. The "art of medicine" still plays an important part in everyday practice, but its focus is evolving. The "art of medicine" is not about applying anecdotal experiences to the solution of clinical problems, it is about critically appraising the evidence in front of us and linking it to our focus of interest, the individual patient.

MARIA E SUAREZ-ALMAZOR ANTHONY S RUSSELL

University of Alberta, Edmonton, Alberta, Canada

Correspondence to: Dr A S Russell, Rheumatology/Clinical Immunology, University of Alberta, 562 Heritage Medical Research Centre, Edmonton, Alberta T6G 2S2, Canada.

1 Brook RH. Practice Guidelines and practising medicine. Are they compatible. JAMA 1989;262:3027-30.

2 Grimshaw J, Russell I. Effect of clinical guidelines on medical practice: a systematic review of rigorous evaluations. Lancet 1993;342:1317-22.

3 Toepp MC, Kuznets N. Directory of practice parameters. Chicago: American Medical Association, 1995.

4 Guidelines for Canadian Clinical Practice Guidelines. Ottawa: Canadian Medical Association, 1994.

5 Kremer JM, Alarcon GS, Lightfoot RW Jr, et al. Methotrexate for rheumatoid arthritis. Suggested guidelines for monitoring liver toxicity. American toid arthritis. Suggested guidelines for monitoring liver toxicity.

6 Hochberg MC, Altman RD, Brandt KD, et al. Guidelines for the medical management of osteoarthritis. Part I. Osteoarthritis of the hip. American management of osteoarthritis. Part I. Osteoarthritis of the hip.
College of Rheumatology. Arthritis Rheum 1995;38:1535-40.

7 Hochberg MC, Altman RD, Brandt KD, et al. Guidelines for the medical management of osteoarthritis. Part II. Osteoarthritis of the knee. American College of Rheumatology. Arthritis Rheum 1995;38:1541-6.
8 American College of Rheumatology Ad Hoc Committee on Clinical Guidelines. Guidelines for the management of rheumatoid arthritis. Arthritis Rheum 1996;39:713-22.

9 Anonymous. Guidelines for monitoring drug therapy in rheumatoid arthritis. American College of Rheumatology Ad Hoc Committee on Clinical Guidelines. Arthritis Rheum 1996;39:723-31.

10 American College of Rheumatology Task Force on Osteoporosis Guidelines. Recommendations for the prevention and treatment of glucocorticoidinduced osteoporosis. Arthritis Rheum 1996;39:1791-801.

11 American College of Rheumatology Ad Hoc Committee on Clinical Guidelines. Guidelines for the initial evaluation of the adult patient with acute musculoskeletal symptoms. Arthritis Rheum 1996;39:1-8.

12 Galindo-Rodriguez G, Avina-Zubieta A, Fitzgerald A, LeClerq S, Russell A, Suarez-Almazor M. Variations and trends in the prescription of initial second line therapy for patients with rheumatoid arthritis. J Rheumatol 1997; 24:633-8

13 Criswell LA, Redfearn WJ. Influence of characteristics of rheumatologists and their practices on the use of prednisone and second-line agents for RA. J Rheumatol 1995;22:829-35.

14 Coyte PC, Hawker G, Croxford R, Attard C, Wright JG. Variation in rheumatologists' and family physicians' perceptions of the indications for and
outcomes of knee replacement surgery. J Rheumatol 1996;23:730-8.

15 Hochberg MC, Perlmutter DL, Hudson JI, Altman RD. Preferences in the management of osteoarthritis of the hip and knee: results of a survey of community-based rheumatologists in the United States. Arthritis Care Res 1996;9:170-6.

16 Cherkin DC, Deyo RA, Wheeler K, Ciol MA. Physician variation in diagnostic testing for low back pain. Who you see is what you get. Arthritis Rheum 1994;37:15-22.

17 Von Korff M, Barlow W, Cherkin D, Deyo R. Effects of practice style in managing back pain. Ann Intern Med 1994;121:187-95.

18 Carey TS, Garrett J. Patterns of ordering diagnostic tests for patients with acute low back pain. The North Carolina Back Pain Project. Ann Intern Med 1996;125:807-14

19 McDonald CJ. Medical heuristics: The silent adjudicators of clinical practice. Ann Intern Med 1996;124:56-62.

20 Tunis SR, Hayward RSA, Wilson MC, Rubin HR, Bass EB, Johnston M, et al. Internists' attitudes about clinical practice guidelines. Ann Intern Med 1994;120:956-63.

21 Hayward RSA, Guyatt GH, Moore KA, McKibbon A, Carter AO. Canadian physicians' attitudes about and preferences regarding clinical practice guidelines. Can Med Assoc J 1997;156:1715-23.

22 DL Sackett, Oxman AD, eds. The Cochrane collaboration handbook. Oxford: Cochrane Collaboration, 1995.

23 Felson DT, Anderson JJ, Meenan RF. Use of short-term efficacy/toxicity tradeoffs to select second-line drugs in rheumatoid arthritis. A meta-
analysis of published clinical trials. Arthritis Rheum 1992;35:1117-35.

24 Rheumatoid Arthritis Clinical Trial Archive Group (RACTAG). The effect of age and renal function on the efficacy and toxicity of methotrexate in rheumatoid arthritis. J Rheumatol 1995;22:218-23.

25 Emery P. Therapeutic approaches for early rheumatoid arthritis. How early? How aggressive? Br J Rheumatol 1995;34 (suppl 2):87-90.

26 Suarez-Almazor ME, Soskolne CL, Saunders LD, Russell AS. Use of second-line drugs in the treatment of rheumatoid arthritis in Edmonton, Alberta: patterns of prescription and long-term effectiveness. J Rheumatol 1995;22:836-43.

27 Eliason BC, Lofton SA, Mark DH. Influence of demographics and profitability on physician selection of family practice procedures. J Fam Pract 1994:39:341-7.

28 Worrall P, Chaulk P, Freake D. The effects of clinical practice guidelines on patient outcomes in primary care: a systematic review. Can Med Assoc J 1997; 156:1705-12.

29 Oxman AD, Thomson MA, Davis DA, Haynes RB. No magic bullets: a systematic review of 102 trials of interventions to improve professional practice. Can Med Assoc J 1995;153:1423-31.

30 Haynes RB, Sackett DL, Muir Gray JA, Cook DL, Guyatt GH. Transferring evidence from research into practice: 2 . Getting the evidence straight. ACP J Club 1997;A14-6.

31 Antman EM, Lau J, Kupelnick B, Mosteller F, Chalmers TC. A comparison of results of meta-analyses of randomized control trials and recommendations of clinical experts. JAMA 1992;268:240-8.

32 Bigos S, Bowyer O, Braen G, Brown K, Deyo R, Haldeman S, et al. Acute low back problems in adults. Clinical practice guideline no 14. AHCPR Publication no 95-0642. Rockville, MD: Agency for Health Care Policy and Research, Public Health Service, US Department of Health and Human Services, 1994

33 Suarez-Almazor ME, Belseck E, Russell AS, Mackel JV. Use of lumbar radiographs for the early diagnosis of low back pain by family physicians. Proposed guidelines would increase utilization. JAMA 1997;277:1782-6.

34 Rogers EM. Diffusion of innovations. 3rd ed. New York: The Free Press, 1983.

35 Russell I, Grimshaw J. The effectiveness of referral guidelines: a review of the methods and findings of published evaluations. In: Roland M, Coulter A, eds. Hospital referrals. Oxford: Oxford University Press, 1992: 179-211.

36 Drummond M, Bosi-Ferraz M, Mason J. Assessing the cost-effectiveness of NSAIDs: an international perspective. J Rheumatol 1995;22:1408-14. 